\title{
Cloning and Expression Analysis of a Novel Mouse Zinc Finger Protein Gene Znf313 Abundantly Expressed in Testis
}

\author{
Na Li, Huaqin Sun, Qiaqing Wu, Dachang Tao, Sizhong Zhang and Yongxin Ma* \\ Department of Medical Genetics, West China Hospital; Division of Human Morbid Genomics, \\ State Key Laboratory of Biotherapy of Human Diseases, Sichuan University, Chengdu, 610041, P. R. China
}

Received 31 October 2006, Accepted 30 November 2006

\begin{abstract}
We have cloned a novel mouse zinc finger protein gene Znf313 by rapid amplification of cDNA ends (RACE) according to the homologue of human $Z N F 313$ gene. The cDNA is 2,163 base pairs (bp) in length and encodes a 229 amino acids (aa) protein with a $\mathrm{C}_{3} \mathrm{HC}_{4}$ ring finger domain and three $\mathrm{C}_{2} \mathrm{H}_{2}$ domains. $89 \%$ and $93 \%$ nucleotide (nt) and aa sequence identity is observed with its human homologue. Revealed by Northern blot and RT-PCR, full mRNA consists of $2.16 \mathrm{~kb}$ and widely expresses in tissues as a single transcript, most abundantly in heart, liver, kidney and testis. The expression of $Z n f 313$ in testis is detected in all development stages. Western blot analysis also reveals that Znf313 is expressed in the tissues. Immunohistochemical staining and subcellular localization demonstrate that $Z n f 313$ is expressed both in the cytoplasm and nucleus whereas predominantly localized in the nucleus. Present data suggests that $\mathrm{Znf313}$ gene might play a fundamental role in gene transcription and regulation in organism and relates to spermatogenesis.
\end{abstract}

Keywords: Mouse Znf313 gene, Northern blot, RACE, RTPCR, Subcellular localization

Spermatogenesis is a complex multistep process requiring highly regulated expression of numerous genes, which located on both autosomes and sex chromosomes (Willison and Ashworth, 1987; Hecht, 1995; Sassone-Corsi, 1997; Vogt, 1998). The products of many genes are essential for spermatogenesis, but only a few of them affect spermatogenesis exclusively. Identification of these genes and their role is important for understanding the biology of spermatogenesis (Cooke et al., 1998).

*To whom correspondence should be addressed.

Tel: 86-28-85164010; Fax: 86-28-85164009

E-mail: mayongxing@263.net or mayongxin.2006@yahoo.com.cn
Zinc finger gene family is the largest human gene family and plays an important role in the regulation of transcription (Free mont, 1993; Cooke and Schwabe, 1995). This large family may be divided into many subfamilies such as Cys2/ His2 type glucocorticoid receptor, ring finger, GATA-1 type, GAL4 type, and LIM family (Barlow et al., 1994; Borden and Freemont, 1996; Hammarstrom et al., 1996). Up to now, more than 25 zinc finger genes have been reported to be involved in spermatogenesis. Some of them are supposed to participate in the transcriptional regulation of spermatogenesis including the genes ZNF76, Sperizin, ZFY, ZFX, mZNF8 and Zfp35 (Cunliffe et al., 1990; Palmer et al., 1990; Ragoussis et al., 1992; Fujii et al., 1999; Jiao et al., 2002), which are usually expressed ubiquitously and function primarily during meiosis and early spermatid development.

While both $\mathrm{C}_{2} \mathrm{H}_{2}$ and ring finger structure are the most extensive and common types of the zinc-binding motif, the two kinds of zinc finger structural domains exist seldom in one protein simultaneously, whereas ZNF313 protein has both of them simultaneously. Human ZNF313 is highly expressed in testicular tissues of fertile adults (Ma et al., 2003). Using homology analysis and rapid amplification of cDNA ends (RACE), we isolate a novel mouse zinc finger protein gene $\mathrm{Znf313}$, the homologue of the human ZNF313 gene, which also contains both $\mathrm{C}_{2} \mathrm{H}_{2}$ and ring finger domain and analyze its expression in many tissues even in early embryo by Northern blot and RT-PCR. Immunohistochemical staining and subcellular localization protocol is used for inspecting the space of its expression product. Summarily, studies on the structure and function of mouse $\mathrm{Znf3} 33$ will provide a clue to understand the molecular mechanism of spermatogenesis.

\section{Materials and Methods}

RNA extraction. Total RNAs from mouse testis and other somatic tissues were prepared by using RNeasy mini Kit (QIAGEN) with DNaseI (RNase-free) to eliminate DNA contamination. Pellets of 
the total RNA were resuspended in DEPC-treated water and stored at $-80^{\circ} \mathrm{C}$ until to use.

Molecular cloning of full-length cDNA of $Z$ nf3 13. The amino acid sequence of human ZNF313 (GenBank Accession No. $A F 265215$ ) was used to search the mouse expressed sequence tag (EST) database at GenBank (http://www.ncbi.nlm.nih.gov). The highly conserved ESTs were used to assemble into a 1,543 bp contig (www.Tigem.it/ESTmachine). Primers for 5' and 3' RACE and the other four pairs of primer to obtain the complete sequence were designed according to the mouse ESTs and human ZNF313 cDNA. 5' and 3'-RACE experiments were performed using SMART RACE cDNA Amplification Kit (Clontech) (Frohman et al., 1998). cDNAs were reverse-transcribed from mouse testis total RNAs templates. The PCR products including RACE products were cloned into the pGEM-T Easy Vector (Promega) and sequenced bidirectionally. Sequences obtained by 5' and 3'-RACE and the other 4 fragments were assembled by removing the overlapping sequence. The assembled full-length cDNA of Znf313 was queried to the mouse genome database (http://www.ncbi.nlm.nih.gov/genome/seq/ MmBlast.html) to determine its chromosomal location and analyze its genomic structure. The deducible amino acid sequence was searched against InterPro database (http://www.ebi.ac.uk/interpro/) for possible functional domains.

Genomic structure analysis. Exon-intron boundaries of the mouse Znf313 gene were identified by aligning the cDNA sequences with corresponding genomic sequence (GenBank accession No. AL589870).

Northern blot analysis. Expression analysis of the $Z n f 313$ transcript was performed using the Multiple Tissue Northern (MTN) Blot Membranes (Clontech cat\# 7762-1) with mRNA from 8 mouse tissues. The cDNA probe for $Z n f 313$ was synthesized from a clone contained $Z n f 313$ gene insertion and was labeled with $\left[{ }^{32} \mathrm{P}\right] \mathrm{dCTP}$ with Random Primed DNA Labeling kit (Roche) according to the manual's protocol and purified with NucleoTrap PCR Purification Kit (Clontech). Northern hybridization was then performed as described by the manual and mouse $\beta$-actin as control.

RT-PCR analysis. Total RNAs from mouse tissues including testis, placenta, heart, eye, brain, skeletal muscle, lung, liver, spleen, kidney, stomach and small intestine were extracted and the other total RNA from mouse testis including: the embryo mice at day 6 , 11 , the fetal mice 2 days prenatal, the newborn mice at day $3,6,10$, 14 , and 21 postnatal (pn), and the adult mice were collected for RTPCR analysis. Total RNAs $(2 \mu \mathrm{g})$ from each sample were reversetranscribed using random primer as primer and Superscript II Reverse Transcriptase (Gibco BRL). Then cDNAs $(2 \mu \mathrm{l})$ were used as template for subsequent PCR experiments. The primers used to amplify a 364 bp fragment of Znf313 were 5'-CTG AAG CCG AAG AAA CCT-3' (forward) and 5'-AGG CAC AGA TGG GAC AAA-3' (reverse). A 517 bp fragment of $\beta$-actin was co-amplified as an internal control. All primers used in RT-PCR were located in different exons. The PCR reaction profile was $94^{\circ} \mathrm{C}$ for $2 \mathrm{~min}$ followed by $94^{\circ} \mathrm{C}$ for $30 \mathrm{~s}, 59^{\circ} \mathrm{C}$ for $30 \mathrm{~s}$ and $72^{\circ} \mathrm{C}$ for $30 \mathrm{~s}$ for 34 cycles, with a final extension stage at $72^{\circ} \mathrm{C}$ for $5 \mathrm{~min}$. The PCR products were then separated by $2 \%$ agarose gel electrophoresis and analyzed the gene expression.
Preparation of polyclonal antibody. A plasmid carrying the human ZNF313 gene was used as the template for the amplification of ZNF313 open reading frame. A 724-bp fragment encoding 228 aa of ZNF313 was inserted into the prokaryotic expression plasmid pET32a (Novagen). An aliquot (12 $\mu \mathrm{l})$ of the products was used to transform $200 \mu \mathrm{l}$ Escherichia coli strain Top10 supercompetent cells according to the standard protocol for rapid screening by restriction map and then the correct recombinant plasmids were transformed to the expression host $E$. coli BL21(DE3).

The purified human $\mathrm{ZNF} 313$ protein $(15 \mathrm{mg})$ was mixed with Freund's complete adjuvant (Sigma) and then immunized two New Zealand white rabbits according to standard protocols. One week after the final immunization, the antibody titer was confirmed. The antiserum was separated by centrifugation at $1,000 \mathrm{~g}$ for $30 \mathrm{~min}$ and dispensed into aliquots, stored at $-70^{\circ} \mathrm{C}$.

Western blot analysis. The tissues from mouse testis, heart, liver, kidney, stomach and brain were homogenized in the lysis buffer $(0.1 \mathrm{~mol} / \mathrm{l} \mathrm{NaCl}, 0.01 \mathrm{~mol} / 1 \mathrm{Tris}-\mathrm{HCl}, 0.001 \mathrm{~mol} / 1$ EDTA), boiled for $8 \mathrm{~min}$ and centrifuged briefly. The homogenized extracts were electrophoresed on an SDS-polyacrylamide gel. Then the proteins were transferred to a polyvinylidene difluoride (PVDF) membrane (Amersham Biosciences). The membrane was incubated with the anti-ZNF313 antibody at a dilution of $1: 800$ for $2 \mathrm{~h}$ and then washed and exposed to horseradish peroxidase-conjugated goat anti-mouse IgG antibodies $(1: 10,000)$ for $1 \mathrm{~h}$. Detection was performed using ECL Western blotting detection reagents (Amersham Biosciences) and finally exposed to FUJI MEDICAL X-RAY FILM (Fujifilm).

Immunohistochemical staining analysis. A labeled-streptoavidinbiotin-peroxidase (LSAB) method was employed for immunohistochemical staining. First, paraffin-embedded tissues of mouse testis were sectioned $(4 \mu \mathrm{m})$. The sections were deparaffined as usual and treated with $3 \%$ cold hydrogen peroxide to suppress endogenous peroxidase activity. Then sections were washed with PBS, incubated with 3\% normal goat serum (Zhongshan Bio.) for $20 \mathrm{~min}$, washed, incubated with the anti-ZNF313 antibody at a dilution of $1: 500$ for $3 \mathrm{~h}$. The sections were then washed, incubated with biotinylated goat anti-rabbit IgG antibody for $30 \mathrm{~min}$, washed and incubated for $30 \mathrm{~min}$ with avidin-biotin linked peroxidase complexes. After washing the sections were treated with streptoavedin peroxidase for 30 min, washed. DAB was applied for 10 min followed by further washing in running water. The slides were then counterstained with dilute aqueous hematoxylin, dehydrated in graded alcohols, cleared in xylene and coverslipped. Most specimens were stained more than once, and essentially identical results were obtained. The resulting stainings were evaluated on an Olympus microscope.

Subcellular localization analysis. To visualize the subcellular localization of the mouse Znf313 protein, we constructed a chimeric protein of Znf313 with green fluorescent protein (GFP), the coding sequence of these cDNA was inserted into pEGFP-N1 plasmid vector (Clontech) in frame.

Nucleotide sequences of the expression vector (pZnf313-EGFP) were finally confirmed by sequencing. For microscopic analysis, HEK293T cells and 3T3 cells were seeded on 6-well slides (Cultureslide, Falcon) and grown in Dulbecco's modified Eagle's medium (Gibco BRL) supplemented with $10 \%$ fetal bovine serum 
(Gibco BRL) and $1 \%$ penicillin-streptomycin (Gibco BRL) at $37^{\circ} \mathrm{C}$ with $5 \% \mathrm{CO}_{2}$. Cells were transfected with $3 \mu \mathrm{g}$ each of plasmid DNA of pZnf313-EGFP or pEGFP-N1 (control) using Lipofectinamine 2000 (Invitrogen) according to the manufacturer's instructions until cells were grown to approximately $70 \%$ confluence in each dishes. Subcellular localization of the fusion proteins were detected $48 \mathrm{~h}$ later by an Olympus fluorescence microscope.

\section{Results}

Cloning and bioinformatics analysis of the full-length cDNA of Znf313. We cloned mouse Znf313 based on the amino acid sequence of human ZNF313 by searching the mouse expressed sequence tag (EST) database from GenBank (http://www.ncbi.nlm.nih.gov) and RT-PCR including RACE. Therefore, two primers for RACE and four pairs of primer were designed based on the homologous ESTs and human ZNF313 cDNA. As a result, six fragments were obtained and cloned into pGEM-T Easy vector and sequenced bi-directionally. After removing the overlapping sequence, full length 2,163 bp cDNA was obtained and submited in the GenBank with the accession number: AF502145.

The predicted open reading frame from 24 to 710 is $687 \mathrm{bp}$ in length that encodes a polypeptide of 229 amino acid residues (Fig. 1). As expected, a canonical polyadenylation signal AATAAA was found $22 \mathrm{bp}$ upstream of the poly (A) tail. It encodes a polypeptide of 229 amino acid residues with a $\mathrm{C}_{3} \mathrm{HC}_{4}$ zinc finger domain from 30 to 68 amino acid residue and three non-standard $\mathrm{C}_{2} \mathrm{H}_{2}$ zinc finger domains, the first one from 90 to 111, the second one from 142 to 165 and the last one from 172 to 200 amino acid residues.

Comparing its nt and aa sequences with those of human homolog, $89 \%$ and $93 \%$ identity was observed respectively. Furthermore, it is remarkable that the aa of ring finger domain and $\mathrm{C}_{2} \mathrm{H}_{2}$ are highly conserved (Fig. 2). Aligned aa sequence of ring finger domain of $\mathrm{Znf} 313$ with those of other mammalian species indicated that this domain is highly conserved. The
91 24

18 54

271

84

361

114

451

144

541

174

631

204

72

81

901

99

1081 AT AGC AC AATAAAGCAGGG ACTCCTTTCCTTGGCGCTGAGGTGCCTTGGTTC TGC AG TTT ACTGGGCGAGGGCTC TGCTCCTCTCCTCCT 1170

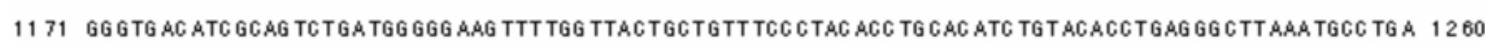

1261 TGGTGCCTTTTTAGGATAAGGTATC ACCATAGAATTG TAGTG GAAATGTTGGGTAGCAAAGATGGGGTG TGTTCATGTGTAAGTAGGGAG 1350

1351 AAGGTGTAGGGTGTGTG GC TTTTTG GTTTAC TTTGATGC TGATGAGTGTTCTCAGGTGGC TGGGTGGTG TGC CGC TC TTATTGAGTATAT 1440

1441 GCTGACATTCTTGCTCTGAAGAGTGTTGGAGGGAGGCACGGACTGCTGGGTGCTGCTTGGTAACTTGATGAGCCTGAGCAGTATTGCTGC 1530

1531 CTCCTTGTGGAAGTATG TTTTAAACCCTGCCTTTTGTTGTGGAGGCAGTGGTAAAGG CAGGGTCATAGC TGGGAAGTTAAAGTTAGGGCC 1 620

1621 CTGCACAGTGGAGGGAG GG GGTCTGCTGAGGCTTTCTTG AGG AGCAGCTATAAGAGC TGGAAATGCAGTCATGTTACAGAGCACAAGGAA 1710

1711 GAGCACC TCCAGTC AGTTC ACAGGACAGAC CCTCTAGC AGGCCTGGGGCTTGGGAG TGAGTC TTC TTTCTGGTCATGGGGCTCTGGTCC 1800 1801 ATCTG AG ACTGCATGTAAC ATTGTACTTGTGGCACCAGGCCTCGACAGTGGTGGCTC TGCCTGGCAGGGCCAAGCCAGCGCAGATGCCAG 1890 1891 CAGAG TCGGAC AGGATC AT TTTCCC AAC GTTACTGCTTC TTC ACTGTACCCTCTG GAAAG GTTTGC TCAGGAGATGTGTGGAG GGAGGTT 1 980 1981 CAACAAG ACCT GCC CCG AGCAACTG GGAAAG GCACAGGTGTGCCACAGTGCAGCAGG GACCTC TTG TCC TTC ATC AC ACTCGCTGTT TCT 2070 2071 AATATGACTTTCGAGAAAG TGTGGTTATGTTCTG TCTTATAG AAAGTGAAATATAAAT MAATT TC ATAATTTATCC AACC AGTAABAAAA 21 G0 $2161 \mathrm{AAB}$

Fig. 1. Nucleotide and predicted amino acid sequence of $Z n f 313$ gene The translation initiation codon is underlined. The stop codon at the 3 '-end of the sequence is underlined and shaded. The predicted $\mathrm{C}_{3} \mathrm{HC}_{4}$ zinc finger motif is boxed and the $\mathrm{C}_{2} \mathrm{H}_{2}$ zinc finger motifs is boxed and shaded. The proximal and the distal polyadenylation signal sequence are bolded. 


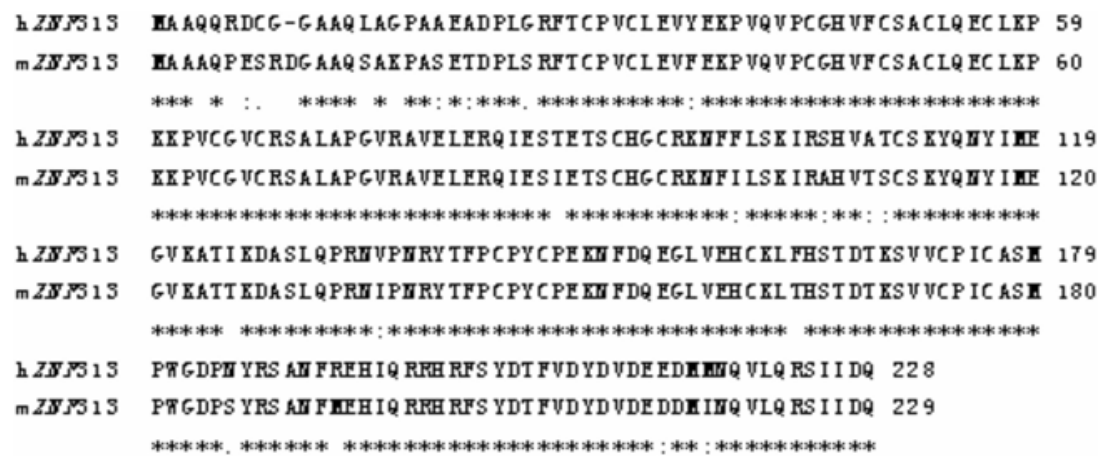

Fig. 2. Alignment of human ZNF313 and mouse Znf313 amino acid sequences. (*) identical or conserved residues; (:) Conserved substitutions; ( . ) semi-conserved substitutions.

conserved primary structure suggests, at least in some extent, that its function may also be conserved and important.

Genomic structures of mouse $Z$ nf313. The human $Z N F 313$ had been mapped on 20q13. After searching the human-mouse homology map (http://www.ncbi.nlm.nih.gov/Homology/), the mouse Znf313 was mapped on chromosome 2. To confirm the localization, the mouse genome database was searched with the cDNA of Znf313. As a result, a contig (AL589870) from chromosome 2 was found. Further analysis revealed that Znf313 spanned 12,736 bp genomic sequences and the cDNA was split into 6 exons. The splicing sites of intron-exon boundaries were conformed to the standard gt/ag rule (Table 1).

\section{The Znf313 gene message is expressed ubiquitously.} Northern blot analysis of mouse eight tissues (Clontech cat\# 7762-1) was used to characterize the expression profiles of the $\mathrm{Znf313}$ gene in various tissues (Fig. 3A). The $Z n f 313$ probe detected a $2.16 \mathrm{~kb}$ transcript in all the tissues examined. But the transcript is expressed highly in heart, liver, kidney and testis, but at a significantly reduced level in brain, spleen, lung. For further determining the Northern results, multiple tissues RT-PCR with total RNAs from mouse testis, placenta, heart, eye, brain, skeletal muscle, lung, liver, spleen, kidney, stomach and small intestine were performed. The expected 364 bp PCR product of the Znf313 gene was ubiquitously expressed in all tissues and the same as the Northern results, strong level signals were detected in heart, liver, kidney and testis whereas a significantly reduced in brain, spleen and lung. As an internal control, mouse $\beta$-actin was expressed equally in all the tissues tested (Fig. 3B).

When we searched against UniGene database with cDNA of Znf313 gene, a UniGene cluster (Hs.10590) was obtained. The cluster included 434 ESTs which came from testis, heart, brain, lung, prostate, kidney as well as some other tissues used or not used in our expression studies. Our results of Northern and RT-PCR analyses were consistent with the UniGene database.

In addition, we also interested in what rules the expression of the mouse Znf313 gene has during development and which stage of spermatogenesis it is expressed. So we collected other total RNA mouse testis including the embryo mice at day 6 , 11 , the fetal mice 2 days prenatal, the newborn mice at day 3 , $6,10,14$, and 21 postnatal (pn), and the adult mice for RTPCR analysis. As shown in Fig. 3C, the expression of Znf313 can be detected in all stage even in early embryo stage. This means that the gene is expressed in early stage of individual development when the spermatogenesis does not occur.

To determine the expression of mouse $\mathrm{Znf} 313$ protein in mouse tissues, we isolated total proteins from various mouse tissues. Western blot analysis indicated that $\mathrm{Znf} 313$ was expressed in the mouse testis, heart, liver, kidney, stomach and

Table 1. Genomic structure of the mouse Znf313 gene

\begin{tabular}{|c|c|c|c|c|c|}
\hline \multirow{2}{*}{ Exon number } & \multirow{2}{*}{ Size (bp) } & \multicolumn{2}{|c|}{ Sequence at intron-exon junction } & \multirow{2}{*}{-Intron number } & \multirow{2}{*}{ Size $(b p)$} \\
\hline & & Splice acceptor & Splice donor & & \\
\hline 1 & 166 & & GCGGACACGTgtgagtggcggg & 1 & 3445 \\
\hline 2 & 151 & gtcttgttctagCTTTTGCTCT & CCGTAAGAATgtatgtggctt & 2 & 3678 \\
\hline 3 & 107 & tttctattcaagTTCATCCTAT & TTCAGCCGAGgtaccgacctac & 3 & 229 \\
\hline 4 & 115 & ctcacttcccagAAACATCCCAA & CAAGTCTGTGgtaagaattggc & 4 & 1190 \\
\hline 5 & 108 & tcctcctgacagGTTTGTCCCA & CACTTTTGTGgtgagtcagcac & 5 & 2041 \\
\hline 6 & 1506 & tcctcetgacagGTTTGTCCCA & & & \\
\hline
\end{tabular}

The splice acceptor/donor columns show sequences that span splicing junctions. The exonic sequences are in upper case, and the intron sequences in lower case. Canonical nucleotides (gt/ag) are in bold type. 


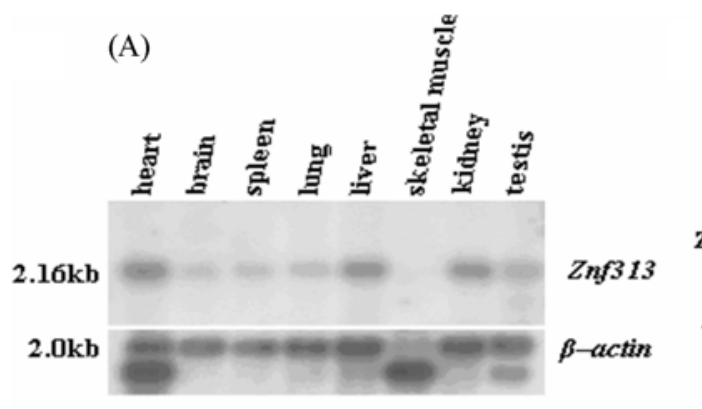

(D)
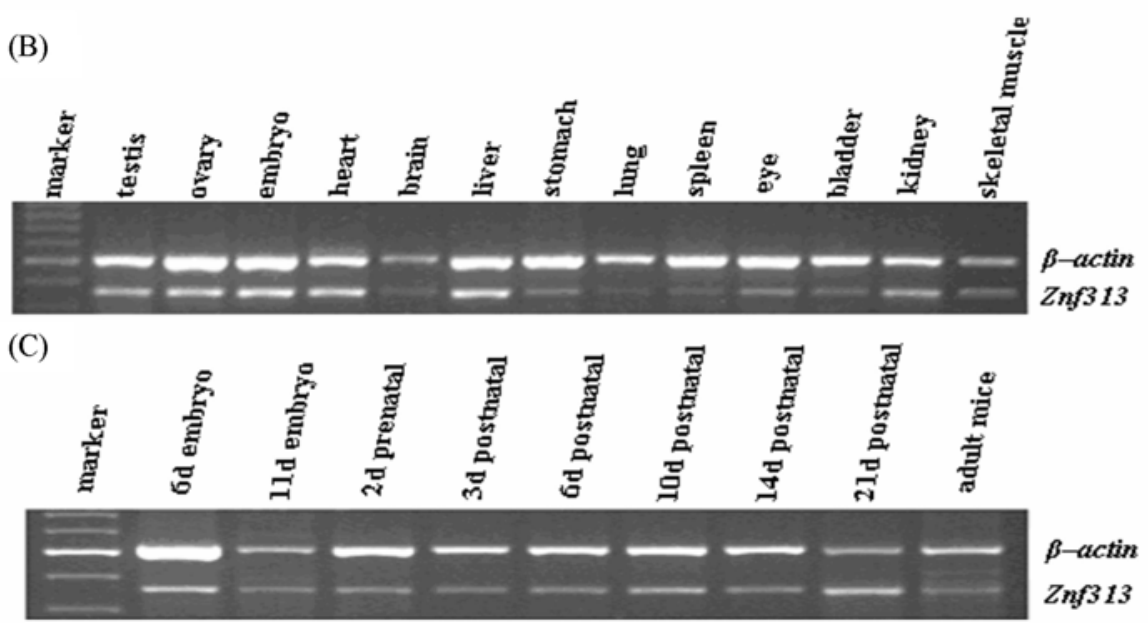

Fig. 3. Expression analysis of $Z n f 313$ in multiple mouse tissues by Northern blot (A), multiple tissues and different developmental stage by RT-PCR $(B, C)$ and multiple tissues by Western blot (D). (A) One transcript was detected, the $2.16 \mathrm{~kb}$ transcript was expressed highly in heart, liver, kidney and testis, but at a significantly reduced level in brain, spleen, lung. mouse $\beta$-actin as a control. (B) The expected $364 \mathrm{bp}$ PCR product of the Znf313 gene was ubiquitously expressed in all tissues but a strongly level signal was detected in heart, liver, kidney and testis whereas a significantly reduced in brain, spleen and lung. (C) RNAs from the embryo mice at day 6 , 11, the fetal mice 2 days prenatal, the newborn mice at day 3, 6, 10, 14, and 21 postnatal (pn), and the adult mice. The expression of Znf313 can be detected in all stage even in early embryo stage. In both (B) and (C), Marker is 100 bp DNA ladder (Takara). The mouse $\beta$-actin (517 bp) is co-amplified as internal control. (D) The anti-ZNF313 antibody was used to detect a protein of about $26 \mathrm{kDa}$, the expected size for full length $\mathrm{Znf313.} \mathrm{The} \mathrm{actin} \mathrm{was} \mathrm{used} \mathrm{as} \mathrm{a} \mathrm{control.}$

brain, but reduced in brain (Fig. 3D) which agree with the Northern blot results.

\section{Immunohistochemical analysis of Znf313 expression in the} testis. To identify where mouse $\mathrm{Znf} 313$ was expressed in the spermatogenic process, immunohistochemical analysis was carried on tissue section of mouse testis by the anti-ZNF313 antibody. Examination of the mouse testes slides showed that the antibody recognized (germ-cell line cells) spermatogonia, spermatocyte and round spermatid, but no signal was detected in sertoli cell (Fig. 4). The results indicated that mouse Znf313 is mainly expressed in mitosis and meiosis of the spermatogenesis, suggesting that it may play a role during this period.

The subcellular localization of mouse Znf313. We constructed recombinant vectors for $\mathrm{Znf313}$ fused with green fluorescent protein (GFP) and then transfected into HEK293T and 3T3 cells, respectively. The parental vector pEGFP-N1 was also transfected to express EGFP as a control. Examination of cells after transfected $48 \mathrm{~h}$ later by a fluorescent microscope showed that the EGFP was detected both in the cytoplasm and nucleus (Fig. 5A). The pZnf313-EGFP fusion protein was enriched in the nucleus of transfected cells but also dispersed in the cytoplasm (Fig. 5B). These suggest that $\mathrm{Znf} 313$ product exist in all of the cells but predominantly, if not exclusively, localize to the nucleus.

\section{Discussion}

The human ZNF313 gene is a novel spermatogenesis-related zinc finger protein gene that contains both a ring finger and three $\mathrm{C}_{2} \mathrm{H}_{2}$ domains. With Blast analysis, we also found some ESTs from mouse, rat, chicken, dog, pig and cow that share identity more than $80 \%$ with human $Z N F 313$ gene. The presence of these homologous and their conservation in evolution suggest that ZNF313 may be important for these organisms.

Revealed by Northern blot and reverse transcriptase polymerase chain reaction (RT-PCR), the mouse Znf313 full mRNA consists of $2.16 \mathrm{~kb}$ and it is widely expressed in 


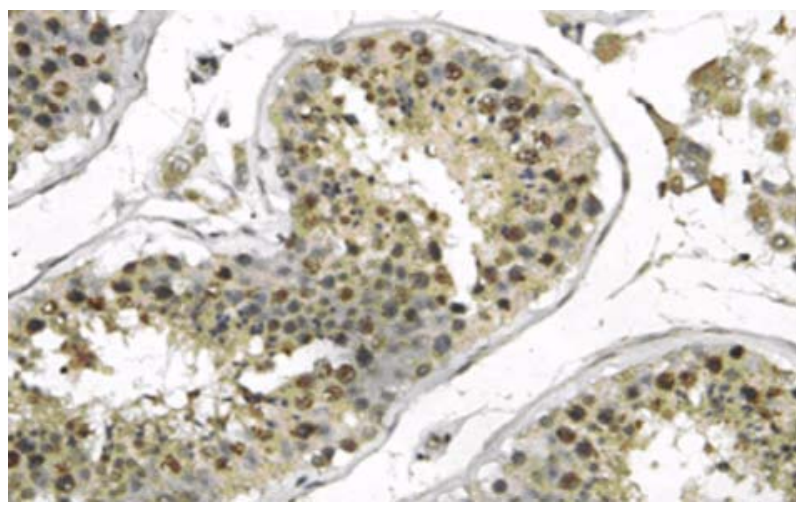

Fig. 4. The location pattern of the $\mathrm{Znf} 313$ protein in mouse testis. The immunohistochemical analysis of $\mathrm{Znf3} 13$ expression in mouse testis revealed that the protein was localized mostly in the nuclear of the germ cell and with faintness signal in the cytoplasm.

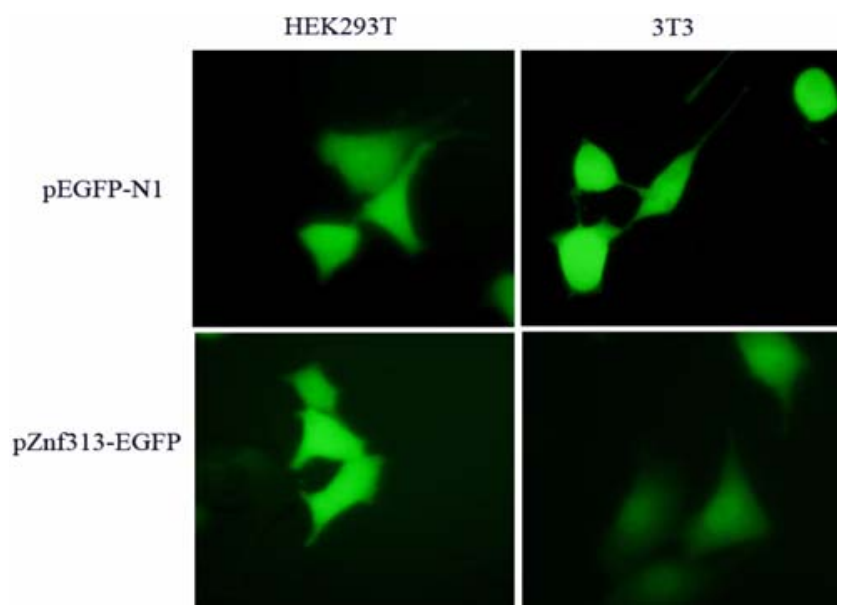

Fig. 5. Subcellular localization analysis of $\mathrm{Znf} 313$ gene products in HEK293T or 3 T3 cells. pEGFP-N1 vector (GFP) as control. pZnf313-EGFP fusion protein. HEK293T or 3T3 cells were transfected with pZnf313-EGFP, pEGFP-N1 vector (GFP), and analyzed by fluorescence microscope when culturing $48 \mathrm{~h}$.

mouse tissues as a single transcript, most abundantly in heart, liver, kidney, testis and even in embryo. This suggests that Znf313 may play a role in many physiological activities of organism.

The protein predicted from the sequence of the $Z n f 313$ cDNA has features that also suggest it could be a DNAbinding protein, and thus a role as a transcriptional activator or repressor is possible. Factors controlling transcription have been implicated as important for the regulated different function of organism. The $\mathrm{pZnf313-EGFP}$ and fusion protein were enriched in the nucleus of transfected cells but also dispersed a few in the cytoplasm. These suggest that the product of $Z n f 313$ exist in all of the cells but predominantly, if not exclusively, localize to the nucleus as nuclear protein.

The $\mathrm{C}_{2} \mathrm{H}_{2}$ (Krüppel-like) domain is a Cys-X2-4-Cys-X12-
His-X7 type motif and bind an atom of $\mathrm{Zn}$, this region in particular may involve in nucleic acid binding (Klug and Schwabe, 1995). The $\mathrm{C}_{3} \mathrm{HC}_{4}$ zinc finger domain resemble a ringer finger including a C-x-H-x-[LIVMFY]-C-x(2)-C[LIVMYA] type motif. Protein with such structure generally are involved in both protein-DNA and protein-protein interaction. $\mathrm{Znf} 313$ protein contains both three $\mathrm{C}_{2} \mathrm{H}_{2}$ domains and one $\mathrm{C}_{3} \mathrm{HC}_{4}$ zinc finger domain. The open reading frame sequence of mouse $Z n f 313$ gene share identity more than $89 \%$ with human ZNF313 gene. They all suggest that this gene may play an important role in gene transcription and regulation.

At present, many known transcriptional factors are the member of zinc finger protein family and zinc finger protein is related to embryogenesis, cell differentiation and some human diseases (Li et al., 2001). According to the structure and quantity of zinc finger, the zinc finger protein can be divided to two types. The first kind of zinc finger protein contains less than five domain of zinc finger, which always participates in proliferation and differentiation of cell; the second kind have more than five domain of zinc finger, which is known little by human (Zhou et al., 2005). Clearly, ZNF313 belongs to the first kind.

According to the result of immunohistochemical analysis of testis, we have found that the pattern of expression of the Znf313 transcript is correlated with periods of intense gene expression important for the normal progress of spermatogenesis. Analysis by immunohistochemical analysis shows that Expression of Znf313 is in spermatogonia, spermatocyte and round spermatid, stage of spermatogenesis when there are generally high levels of transcription, but no signal was detected in sertoli cell. An inference can be made from these observations, that the expression pattern of Znf313 is what would be expected if its protein product were required for the whole period of transcriptional regulation in spermatogenesis after differentiation from sertoli cell. However, the observation of expression pattern alone cannot demonstrate such a role, and further evidence is needed.

Mouse Znf313 gene is homolog to human ZNF313 gene which is related to spermatogenesis and isolated by Ma et al. (2003). As a possible transcriptional factor, it's intriguing to detect the upstream and downstream gene or factor and the entire signal pathway of spermatogenesis the ZNF313 participates in the future.

Acknowledgments This work was supported by the grant of National Natural Science Foundation of China (No. 90408025, No. 30500186 and No. 30200153 )

\section{References}

Barlow, P. N., Luisi, B., Milner, A., Elliott, M. and Everett, R. (1994) Structure of the C3HC4 domain by ${ }^{1} \mathrm{H}$-nuclear magnetic resonance spectroscopy. A new structural class of zinc-finger. J. Mol. Biol. 237, 201-211. 
Borden, K. L. and Freemont, P. S. (1996) The RING finger domain: a recent example of a sequence-structure family. Curr. Opin. Struct. Biol. 63, 95-401.

Cooke, H. J., Hargreave, T. and Elliott, D. J. (1998) Understanding the genes involved in spermatogenesis: A progress report. Ferti Steril. 69, 989-999.

Cunliffe, V., Koopman, P., McLaren, A. and Trowsdale, J. (1990) A mouse zinc finger gene which is transiently expressed during spermatogenesis. EMBO J. 9, 197-205.

Free mont, P. S. (1993) The ring finger. A novel protein sequence motif related to the zinc finger. Ann. NY Acid Sci. 684, 174192.

Frohman, M. A., Dush, M. K. and Martin, G. R. (1988) Rapid production of full-length cDNAs from rare transcripts: amplification using a single gene-specific oligonucleotide primer. Proc. Natl. Acad. Sci. USA 85, 8998-9002.

Fujii, T., Tamura, K., Copeland, N. G., Gillbert, D. J., Jenkins, N. A., Yomogida, K. and Tanaka, H. (1999) Sperizin is a murine ring zinc-finger protein specifically expressed in haploid germ cells. Genomics 57, 94-101.

Hammarstrom, A., Berndt, K. D., Sillard, R., Adermann, K. and Otting, G. (1996) Solution structure of a naturally-occurring zinc-peptide complex demonstrates that the N-terminal zincbinding module of the Lasp-1 LIM domain is an independent folding unit. Biochemistry 35, 12723-12732.

Hecht, N. B. (1995) The making of a spermatozoon: a molecular perspective. Dev. Genet. 16, 95-103.

Jiao, K., Zhou, Y. and Hogan, B. L. (2002) Identification of $\mathrm{mZNF8}$, a mouse Kruppel-like transcriptional repressor, as a novel nuclear interaction partner of Smad1. Mol. Cell. Biol. 22, 7633-7644.

Klug, A. and Schwabe, J. W. (1995) Protein motifs 5.Zinc fingers. FASEB J. 9, 597-604.

$\mathrm{Li}$, J. Z., Li, J. and Fu, J. L. (2001) Zinc finger protein gene ZNF191. Life of Chemistry 21, 116-117.

Ma, Y. X., Zhang, S. Z., Hou, Y. P. and Huang, X. L. (2003) Indentification of a novel human zinc finger protein gene ZNF313. Acta Bioch. Bioph. Sin. 35, 230-237.

Palmer, M. S., Berta, P., Sinclair, A. H., Pym, B. and Goodfellow, P. N. (1990) Comparation of human ZFY and ZFX transcripts. Proc. Natl. Acid. Sci. USA 87, 1681-1685.

Ragoussis, J., Senger, G., Mockridge, I., Sanseau, P., Ruddy, S., Dudley, K. and Sheer, D. (1992) A testis-expressed Zn finger gene $(Z N F 76)$ in human 6p21.3 centromeric to the MHC is closely linked to the human homolog of the t-complex gene tcp-11.Genomics 3, 673-679.

Sassone-Corsi, P. (1997) Transcriptional checkpoints determining the fate of male germ cells. Cell 88, 163-166.

Vogt, P. H. (1998) Human chromosome deletions in Yq11, AZF candidate genes and male fertility: history and update. Mol. Hum. Reprod. 4, 739-744.

Willison, K. and Ashworth, A. (1987) Mammalian spermatogenic gene expression. Trends Genet. 3, 351-355.

Zhou, C., Li, L. Y. and Lu, G. X. (2005) Molecular cloning and character analysis of the mouse zinc finger protein gene ZFP474 exclusively expressed in testis and ovary. Acta Genetica Sinica. 32, 155-162. 